\title{
Knots not concordant to L-space knots
}

\author{
Ramazan Yozgyur ${ }^{1}$
}

Published online: 30 March 2020

(c) The Author(s) 2020

\begin{abstract}
In this short note we use methods of Friedl, Livingston and Zentner to show that there are knots that are not algebraically concordant to a connected sum of positive and negative L-space knots.
\end{abstract}

Keywords Knots · Concordant knots · L-space knot

\section{Introduction}

A closed oriented 3-manifold $Y$ is called an L-space if it is a rational homology sphere and its Heegaard-Floer homology has minimal possible rank i.e. $\widehat{H F}(Y)=\left|H_{1}(Y ; \mathbb{Z})\right|$. A knot $K \subset S^{3}$ is a called (positive) L-space knot if a surgery with a sufficiently large positive coefficient is an L-space.

L-space knots were introduced by Ozsváth and Szabó in [10] in their attempt to classify knots such that a surgery on them gives a lens space. In particular, they proved the following result.

Theorem 1.1 [10, Corollary 1.3] If $\Delta$ is the Alexander polynomial of an L-space knot, then $\exists a_{1}, \ldots, a_{n}$ such that $a_{0}<a_{1} \ldots<a_{n}$ and

$$
\Delta=t^{a_{0}}-t^{a_{1}}+t^{a_{2}} \cdots+t^{a_{n}}
$$

The class of L-space knots includes all torus knots. More generally, all algebraic knots are L-space knots; see [6,7]. Moreover, in [8] Hedden proves the following result:

Theorem 1.2 An L-space knot is strongly quasipositive and fibred.

We refer to [12] for the definition and the properties of strongly quasipositive knots.

We have the following result of Borodzik and Feller.

Theorem 1.3 (see [1]) Every link L is topologically concordant to a strongly quasipositive link $L^{\prime}$.

The author was supported by the National Science Center Grant 2016/22/E/ST1/00040.

$凶$ Ramazan Yozgyur

ryozgyur@mimuw.edu.pl

1 Institute of Mathematics, University of Warsaw, ul. Banacha 2, 02-097 Warsaw, Poland 
In this light, the main theorem of the paper seems a bit surprising.

Theorem 1.4 There are knots that are not topologically concordant to any combinations of L-space knots and their mirrors.

\section{Proof of Theorem 1.4}

Let us recall the following fact, which can be found e.g. in [11, Section 8.1].

Theorem 2.1 (Cauchy's bound) Suppose $P=\alpha_{0}+\alpha_{1} t+\cdots+\alpha_{m} t^{m}$ is a complex polynomial with $\alpha_{m} \neq 0$.

For any root $z$ of $P$ we have $|z|<1+\max _{j<m} \frac{\left|\alpha_{j}\right|}{\left|\alpha_{m}\right|}$.

From Cauchy's bound we obtain the following proposition.

Proposition 2.2 The Alexander polynomial of an L-space knot has roots with modulus at most 2.

Remark 2.3 From the multiplicativity of the Alexander polynomial under connected sums we infer also that if $K$ is a connected sum of L-space knots and their mirrors, then $\Delta_{K}(t)$ has all roots inside of the disk of radius 2. Note that by [9], a non-trivial connected sum of L-space knots is not an L-space knot anymore.

Following [4], for $n=1,2, \ldots$ we define

$$
P_{n}=1+n t-(2 n+1) t^{2}+n t^{3}+t^{4} .
$$

We need some properties of roots of this polynomial.

Theorem 2.4 (see $\left[4\right.$, Lemma 4]) The polynomial $P_{n}$ is irreducible over $\mathbb{Q}[t]$ and it has two roots on the unit circle.

Denote these two roots by $\theta_{n}^{+}$and $\theta_{n}^{-}$. We will use a more specific control for one of the other roots of $P_{n}$.

Lemma 2.5 For $n \geq 1$, the polynomial $P_{n}$ has a root with modulus greater than 2 .

Proof We have $P_{n}(-n-1)=1-2 n-3 n^{2}-n^{3}<0$. On the other hand, $P(-(n+2))=$ $13+10 n+2 n^{2}>0$. Therefore $P_{n}$ has a real root in the interval $(-n-2,-n-1)$.

Theorem 2.6 Let $K$ be connected sum of L-space knots and some mirrors of L-space knots, then $\Delta_{K}$ cannot vanish at $\theta_{n}^{+}$.

Proof Since the Alexander polynomial of a connected sum of knots is the product of the Alexander polynomial of each knot, it is enough to prove the theorem for $K$ being an Lspace knot.

Suppose $\Delta_{K}\left(\theta_{n}^{+}\right)=0$. Then $\operatorname{gcd}\left(\Delta_{K}, P_{n}\right)$ has positive degree and it divides $P_{n}$. As $P_{n}$ is irreducible over $\mathbb{Q}[t]$, it follows that $P_{n} \mid \Delta_{K}$. But $P_{n}$ has a root outside a disk of radius 2 and all the roots of $\Delta_{K}$ are inside this disk. 
Theorem 2.7 Suppose $K$ is a knot that is concordant to a knot $K^{\prime}$ which is a connected sum of L-space knots and mirrors of L-space knots. Then the order of the root of $\Delta_{K}$ at $\theta_{n}^{+}$is an even number.

Proof As $K$ and $K^{\prime}$ are concordant, an easy corollary of Fox-Milnor theorem [5] implies that there exist polynomials $f, g \in \mathbb{Z}\left[t, t^{-1}\right]$ such that

$$
\Delta_{K}(t) f(t) f\left(t^{-1}\right)=\Delta_{K^{\prime}}(t) g(t) g\left(t^{-1}\right) .
$$

Claim. If $\xi(t) \in \mathbb{Z}\left[t, t^{-1}\right]$ vanishes at $\theta_{n}^{+}$, then $\xi^{-1}(t)$ vanishes at $\theta_{n}^{+}$with the same order.

To prove the claim, note that if $\xi$ vanishes at $\theta_{n}^{+}$up to order $s$, then it is divisible by $\left(t-\theta_{n}^{+}\right)^{s}$ and also by $\left(t-\theta_{n}^{-}\right)^{s}$ (because $\xi$ has real coefficients and $\overline{\theta_{n}^{+}}=\theta_{n}^{-}$). As $\theta_{n}^{+} \theta_{n}^{-}=1$ we have

$$
\left(t^{-1}-\theta_{n}^{+}\right)\left(t^{-1}-\theta_{n}^{-}\right)=t^{-2}\left(t-\theta_{n}^{+}\right)\left(t-\theta_{n}^{-}\right) .
$$

From the above identity the claim follows readily.

From the claim we conclude that the order of the root of $\Delta_{K^{\prime}} g(t) g\left(t^{-1}\right)$ at $\theta_{n}^{+}$is an even number. Using the claim once again, this time for $f(t)$, we see that (2.2) implies that $\Delta_{K}(t)$ vanishes at $\theta_{n}^{+}$up to an even power (maybe zero).

To conclude the proof of Theorem 1.4 we will show that there exist knots such that their Alexander polynomial vanishes at $\theta_{n}^{+}$with an odd order. As $P_{n}$ is a symmetric polynomial and $P_{n}(1)=1$, for any $n$ there exist a knot $K_{n}$ such that $\Delta_{K_{n}}=P_{n}$, see [13]. Furthermore, the knot $K_{n}$ from [4, Figure 1] has Alexander polynomial $P_{n}$.

Example 2.8 A notable example of a knot that is not concordant to a combination of L-space knots is the knot $12 n 642$. According to KnotInfo web page [2], its Alexander polynomial is $P_{7}$. On the other hand, $12 n 642$ is strongly quasipositive and fibered. It is also almost positive in the sense of [3].

Acknowledgements The author is very grateful to his advisor, Maciej Borodzik, for his help during preparation of the manuscript. He also expresses his gratitude towards Chuck Livingston, Stefan Friedl and András Stipsicz for their comments on the preliminary version of the manuscript.

Open Access This article is licensed under a Creative Commons Attribution 4.0 International License, which permits use, sharing, adaptation, distribution and reproduction in any medium or format, as long as you give appropriate credit to the original author(s) and the source, provide a link to the Creative Commons licence, and indicate if changes were made. The images or other third party material in this article are included in the article's Creative Commons licence, unless indicated otherwise in a credit line to the material. If material is not included in the article's Creative Commons licence and your intended use is not permitted by statutory regulation or exceeds the permitted use, you will need to obtain permission directly from the copyright holder. To view a copy of this licence, visit http://creativecommons.org/licenses/by/4.0/.

\section{References}

1. M. Borodzik, P. Feller, Up to topological concordance, links are strongly quasipositive. J. des math. pures et appliquées (2019)

2. J. Cha, C. Livingston, Knotinfo: Table of knot invariants. (2018) Accessed 04 Oct 2019

3. P. Feller, L. Lewark, A. Lobb, Almost positive links are strongly quasipositive (2018). preprint 2018,

4. S. Friedl, C. Livingston, R. Zentner, Knot concordances and alternating knots. Michigan Math. J. 66(2), 421-432 (2017)

5. R.H. Fox, J.W. Milnor, Singularities of 2-spheres in 4-space and cobordism of knots. Osaka J. Math. 3, 257-267 (1966) 
6. E. Gorsky, A. Némethi, Links of plane curve singularities are $L$-space links. Algebraic Geom. Topol. 16(4), 1905-1912 (2016)

7. M. Hedden, On knot Floer homology and cabling. II. Int. Math. Res. Not. IMRN 12, 2248-2274 (2009)

8. M. Hedden, Notions of positivity and the Ozsváth-Szabó concordance invariant. J. Knot Theory Ramif. 19(5), 617-629 (2010)

9. D. Krcatovich, The reduced knot Floer complex. Topol. Appl. 194, 171-201 (2015)

10. P. Ozsváth, Z. Szabó, On knot Floer homology and lens space surgeries. Topology 44(6), 1281-1300 (2005)

11. Q.I. Rahman, G. Schmeisser, Analytic Theory of Polynomials, London Mathematical Society Monographs. New Series, vol. 26 (Oxford University Press, Oxford, 2002)

12. L. Rudolph, Knot theory of Complex Plane Curves, Handbook of Knot Theory (Elsevier B. V, Amsterdam, 2005), pp. 349-427

13. H. Seifert, Über das Geschlecht von Knoten. Math. Ann. 110(1), 571-592 (1935)

Publisher's Note Springer Nature remains neutral with regard to jurisdictional claims in published maps and institutional affiliations. 